\title{
Probable Values of the Cosmological Constant in a Holographic Theory
}

\author{
Petr Hořava ${ }^{1,2, *}$ and Djordje Minic ${ }^{1,3, \dagger}$ \\ ${ }^{1}$ CIT-USC Center for Theoretical Physics \\ ${ }^{2}$ California Institute of Technology, Pasadena, California 91125 \\ ${ }^{3}$ Department of Physics and Astronomy, University of Southern California, Los Angeles, California 90089-0484
}

(Received 25 January 2000)

\begin{abstract}
We point out that for a large class of universes, holography implies that the most probable value for the cosmological constant is zero. In four space-time dimensions, the probability distribution takes the Baum-Hawking form, $d P \sim \exp \left(c M_{p}^{2} / \Lambda\right) d \Lambda$.
\end{abstract}

PACS numbers: 98.80.Cq, 04.70.Dy, 11.10.Kk

1. Introduction. - One of the central problems of theoretical physics is why the cosmological constant is small [1].

The cosmological constant problem has a twofold meaning: It is a problem of fundamental physics, because the value of the cosmological constant $\Lambda$ is tied to vacuum energy density. On the other hand, the cosmological constant tells us something about the large scale behavior of the universe, since a small cosmological constant implies that the observable universe is big and (nearly) flat. The problem is that there is an enormous discrepancy between the value of the vacuum energy density as predicted by quantum field theory of the standard-model degrees of freedom and the cosmologically observed value of $\Lambda$ [2]. This discrepancy occurs already at very low-energy scales, of the order of $\mathrm{eV}$, and clearly represents the most flagrant naturalness problem in today's physics.

Thus, the cosmological constant relates the properties of the microscopic physics of the vacuum to the long-distance physics on cosmic scales. [This general philosophy was stressed in the wormhole approach to the cosmological constant problem (see [3] for the original references and $[4,5]$ for a critique of this approach).] Therefore, the observable smallness of the cosmological constant should tell us something fundamental about the underlying microscopic theory of nature.

In this note we study implications of holography [6,7], taken as a fundamental property of the microscopic theory of quantum gravity, for the cosmological constant problem. Assuming that the cosmological constant is a random variable, and that holographic entropy can be given a Boltzmannian interpretation, we point out that the most probable value of the cosmological constant in a holographic theory is zero, in ensembles of universes with finite-area holographic screens.

It is not the aim of this paper to discuss the microscopic origin of any of these assumptions. In particular, the microscopic origin of holography and the randomness of the cosmological constant are clearly difficult problems whose solutions we do not claim to have understood. It is our goal to show that once these assumptions are satisfied, a simple and robust phenomenological argument implies a naturally small cosmological constant.
2. Holography and the cosmological constant. - It has been suggested on rather general grounds [8-10] that holography should be relevant for solving the cosmological constant problem. In its simplest heuristic form, this argument can be stated as follows. The cosmological constant problem in local quantum field theory is a naturalness problem, following from a gross overcount of the degrees of freedom of the vacuum. In a holographic theory, the Bekenstein-Hawking bound [7] imposes a natural limit on the number of degrees of freedom, which subsequently reduces the vacuum energy density in the microscopic theory. Notice that this intuitive argument does not suggest that $\Lambda$ should be zero; instead, it would make a small but nonzero cosmological constant natural. (Various other aspects of the cosmological constant problem in the light of holography have been recently studied in [11].)

In order to make this argument work on a practical level, we may need a precise microscopic model that exhibits holography in a manifest way. A phenomenological model of holography has been proposed in [9], in the context of $M$ theory. In that model one is led, schematically, to the following expansion of the low-energy effective action in four space-time dimensions

$$
S \approx N M^{2} \int d^{4} x \sqrt{g}\left(M^{2}+R+\frac{1}{M^{2}} R^{2}+\cdots\right)
$$

(in this expansion we ignore various multiplicative constants of the order of 1). (The phenomenological theory in [9] is formulated in eleven space-time dimensions; here we have implicitly compactified the theory to four dimensions on $T^{7}$.) Here $M$ is an infrared scale, essentially the inverse size of the system; $N$ is tied to the number of degrees of freedom in the theory; and $R^{2}$ symbolically denotes the terms quadratic in the Riemann tensor. The low-energy (super)gravity regime is defined as the regime in which the Einstein-Hilbert term is kept finite; this determines the Newton constant $G_{N}$ in terms of the infrared mass $M$ and the number of degrees of freedom $N$

$$
G_{N}{ }^{-1} \approx N M^{2} .
$$

This can be interpreted as the Bekenstein-Hawking formula for $N$ in terms of the area of the surface surrounding 
the system, measured in Planck units. Another indication for holography comes from the following observation. The Einstein-Hilbert term is dominant only if the higherorder curvature terms are suppressed, which happens for $R \ll M^{2}$. This bound coincides with the Bekenstein bound [9]. For exactly the same reason the cosmological term in (1) is naturally small, of the order of $\Lambda \approx M^{2}$ - the "Hubble radius" of the system.

Thus, the phenomenological approach of [9] seems to suggest at a somewhat semiquantitative level that the cosmological constant problem-as a naturalness problem - could indeed be solved in a holographic theory (for other arguments from a different point of view, see [8]). However, the problem of finding a truly microscopic theory that manifestly satisfies holography still remains a fascinating challenge that has not been satisfactorily met so far.

Short of a microscopic formulation of a holographic theory, we choose a different strategy to address the cosmological constant problem. We simply assume that holography is valid, and show that in combination with certain robust thermodynamic arguments, holography indeed implies that the most probable value of the cosmological constant is zero.

For concreteness we work in four space-time dimensions, but the argument can be easily generalized to other cases as well.

At large scales gravity is described by the most general local effective action which incorporates the four-dimensional diffeomorphism invariance (the Einstein-Hilbert action, the cosmological constant term, plus higher-order terms in the curvature tensor and its derivatives)

$$
\begin{array}{r}
S_{\text {eff }}=-\int d^{4} x \sqrt{g}\left(\frac{1}{8 \pi G} \Lambda+\frac{1}{16 \pi G} R+a R_{\mu \nu} R^{\mu \nu}\right. \\
\left.+b R^{2}+c R_{\mu \nu \rho \sigma} R^{\mu \nu \rho \sigma}+\cdots\right) .
\end{array}
$$

Note that $\Lambda$ denotes an effective cosmological constant, which also takes into account the vacuum energy density of matter. We also assume that $\Lambda$ is a random variable, without specifying a particular mechanism that leads to a stochastic $\Lambda$. Several such mechanisms are available in the literature [1]: coupling gravity to a three-form gauge field [12], topology change [3], and chaotic inflation [13] to name a few.

According to the holographic principle [6,14], the entire information about the space-time can be stored on particular hypersurfaces (called holographic screens) [14], such that the total number of degrees of freedom living on these holographic screens does not exceed the BekensteinHawking bound. At present this is the only available formulation of the holographic principle, but it will be sufficient for our argument.
Let us concentrate on a class of universes with welldefined holographic screens of finite area, examples of which were explicitly constructed for various space-time geometries in [14]. In this class of universes, a closer look at Einstein's equations leads to the following scaling relation between the characteristic size $r$ of the preferred screen and the cosmological constant

$$
\Lambda r^{2} \approx 1 \text {. }
$$

As can be seen in [14], this relation between $\Lambda$ and $r$ is valid for a broad class of holographic screens in spacetimes with non-negative $\Lambda$, including the de Sitter spacetime, the Einstein static universe, and a class of the Robertson-Walker cosmologies. In the de Sitter case, for example, the preferred screen is the cosmological horizon [14], whose area is given by [15]

$$
A=\frac{12 \pi}{\Lambda} .
$$

In the anti-de Sitter space-time, the size of the holographic screen is infinite, and we do not know whether our argument can be extended to the cases with holographic screens of infinite area.

The holographic principle asserts that the total number of degrees of freedom, or entropy $S$, living on the holographic screen is bounded by one quarter of the area in Planck units,

$$
S \approx r^{2} M_{p}^{2} .
$$

Of course, this formula is just an upper bound and should be really treated as an inequality. We will take this into account below.

Using the scaling relation (4) valid for the holographic screens, together with the Bekenstein-Hawking bound (6), we get an intriguing formula which relates the cosmological constant and the holographic gravitational entropy $S$

$$
\Lambda S \approx M_{p}^{2} .
$$

More precisely, this formula should be viewed as an inequality

$$
S \leq \frac{M_{p}^{2}}{\Lambda} .
$$

Thus, the holographic gravitational entropy (or the total number of degrees of freedom living on the holographic screens) $S$ increases with a decreasing cosmological constant.

We use this observation to argue that the most probable value for the cosmological constant is zero. The argument proceeds as follows.

At large distances the cosmological constant could be treated as a classical variable (its fluctuations can be neglected). Thus, following the Boltzmann principle from statistical mechanics [16], the probability distribution for the cosmological constant $\Lambda$ to have a value in the interval 
from $\Lambda$ to $\Lambda+d \Lambda$ is given by

$$
w(\Lambda) d \Lambda=\text { const } \times \exp \{S(\Lambda)\}
$$

(with the Boltzmann constant set to 1). In (9), $S(\Lambda)$ is the holographic entropy, which can be formally regarded as a function of $\Lambda$ using (8). We conclude that

$$
w(\Lambda) d \Lambda=\text { const } \times \exp \left\{\frac{c M_{p}^{2}}{\Lambda}\right\} .
$$

Here $c$ denotes a constant of the order of 1 which takes into account the neglected numerical factors. This formula tells us that the probability distribution is strongly peaked around the value $\Lambda=0+$. Hence, the most probable value for the cosmological constant, as implied by holography and thermodynamics, is zero.

We have obtained this formula by saturating the Bekenstein-Hawking bound, but it is easy to see that even if the holographic bound is taken as a true inequality the same conclusion follows, due to the properties of the function $\exp \left\{\left(c M_{p}^{2}\right) / \Lambda\right\}$, and the fact that the probability distributions have to be normalized to 1 . Notice also that we have implicitly worked with a microcanonical ensemble of universes, given our Boltzmannian interpretation of the holographic entropy. (Some aspects of the microcanonical ensemble for gravity have been discussed in [17].)

3. Discussion. - Here we compare our result with the Baum-Hawking mechanism for the vanishing of the cosmological constant.

Our probability distribution (10) exhibits the same exponential dependence on $1 / \Lambda$ as the probability formula obtained in Euclidean quantum gravity by Baum and Hawking [12]. (In $D$ space-time dimensions, our probability distribution generalizes to $\exp \left\{\tilde{c} M_{p}^{D-2} \Lambda^{(2-D) / 2}\right\}$, and therefore gives a functional dependence on $\Lambda$ which again agrees with the result of the Euclidean path integral approach.) In the case of de Sitter universes, the exact value of the numerical constant $c$ in (10) follows from (5), and is found to be $c=3 \pi$. Thus, for de Sitter universes, our holographic probability distribution exactly coincides with the Baum-Hawking distribution.

Recall that the Baum-Hawking mechanism assumes the validity of the Euclidean effective action formalism in quantum gravity; the Minkowski action would lead to an oscillating factor $\exp \left[i\left(M_{p}^{2} / \Lambda\right)\right]$ which completely changes the conclusion about the most probable value for the cosmological constant. Also, the Euclidean action for Einstein's gravity is well known to be unbounded from below; this fact renders the Baum-Hawking mechanism rather problematic. Moreover, it is not clear-at least within the Hamiltonian approach as presented in [5] - whether the Baum-Hawking factor $\exp \left[\left(c M_{p}^{2}\right) / \Lambda\right]$ can be interpreted as a probability distribution in the context of Euclidean quantum gravity with space-time topology change.

By contrast, our argument is based on the assumptions of holography (without specifying its microscopic origin) and thermodynamics. According to the holographic principle, the holographic entropy counts the total number of degrees of freedom in quantum gravity. The use of the Boltzmann formula is therefore justified by the microscopic definition of entropy. Likewise, the use of the problematic Euclidean formalism of quantum gravity has been completely avoided by the use of the holographic principle. Nevertheless, we find it intriguing that the same probability distribution is found in both cases.

In this paper, we have presented a simple argument suggesting that in a holographic theory, the cosmological constant can be naturally small. This conclusion follows from the simple but somewhat subtle fact that by maximizing the entropy in a holographic theory, one minimizes the vacuum energy density. Indeed, small vacuum energy density (hence small $\Lambda$ ) implies a large universe, and therefore more degrees of freedom. As we have argued, this simple correspondence is natural in a holographic theory, yet it is contrary to our experience with conventional local field theory, where large entropy gives a large contribution to the vacuum energy density.

We wish to thank T. Banks, J. Polchinski, A. Strominger, and E. Witten for useful discussions. The work of P. H. has been supported in part by a Sherman Fairchild Prize Fellowship and by DOE Grant No. DE-FG03-92-ER 40701. The work of D. M. has been supported in part by the U.S. Department of Energy under Grant No. DE-FG03-84ER 40168.

*Email address: horava@theory.caltech.edu

${ }^{\dagger}$ Email address: minic@physics.usc.edu

[1] S. Weinberg, Rev. Mod. Phys. 61, 1 (1989); S. M. Carroll, W. H. Press, and E.L. Turner, Annu. Rev. Astron. Astrophys. 30, 499 (1992).

[2] A nice review of recent data is N.A. Bachall, J.P. Ostriker, S. Pelmutter, and P. J. Steinhardt, Science 284, 1481 (1999).

[3] S. Coleman, Nucl. Phys. B307, 867 (1988); B310, 643 (1988); S. Giddings and A. Stominger, Nucl. Phys. B306, 890 (1988); B307, 854 (1988); T. Banks, Nucl. Phys. B309, 493 (1988); T. Banks, I. Klebanov, and L. Susskind, Nucl. Phys. B317, 665 (1989); S. B. Giddings, in Proceedings of the International Colloquium on Modern Quantum Field Theory, Bombay, India, 1990, edited by S. Das et al. (World Scientific, Singapore, 1991).

[4] J. Polchinski, Phys. Lett. B 219, 251 (1989); V. Kaplunovsky (unpublished); W. Fischler and L. Susskind, Phys. Lett. B 217, 48 (1989).

[5] W. Fischler, I. Klebanov, J. Polchinski, and L. Susskind, Nucl. Phys. B327, 157 (1989).

[6] G. 't Hooft, gr-qc/9310026; L. Susskind, J. Math. Phys. (N.Y.) 36, 6377 (1995).

[7] S. W. Hawking, Phys. Rev. D 13, 191 (1976); J. D. Bekenstein, Phys. Rev. D 23, 287 (1981).

[8] T. Banks, hep-th/9601151.

[9] P. Hořava, Phys. Rev. D 59, 046004 (1999).

[10] A. G. Cohen, D. B. Kaplan, and A. E. Nelson, Phys. Rev. Lett. 82, 4971 (1999). 
[11] C.P. Burgess, R.C. Myers, and F. Quevedo, hep-th/ 9911164; H. Verlinde and E. Verlinde, hep-th/9912018; C. Schmidhuber, hep-th/9912156.

[12] E. Baum, Phys. Lett. 133B, 185 (1983); S. W. Hawking, Phys. Lett. 134B, 403 (1984).

[13] A.D. Linde, Phys. Lett. B 175, 395 (1986); 202, 194 (1988).
[14] R. Bousso, hep-th/9905177; hep-th/9906022; hep-th/ 9911002.

[15] G. W. Gibbons and S. W. Hawking, Phys. Rev. D 15, 2738 (1977).

[16] L.D. Landau and E. M. Lifshitz, Statistical Physics (Pergamon, New York, 1988), Pt. 1.

[17] J. D. Brown and J. W. York, Phys. Rev. D 47, 1420 (1993). 